\title{
Grade Management in Establishing Pediatric Peripheral Venous
}

\section{Access}

\author{
Ya-Min Yan, ${ }^{1}$ Mei Gong, ${ }^{2,}$ Dan Li, ${ }^{3}$ Ye Huang, ${ }^{1}$ Ai-Qiu Li, ${ }^{4}$ Jia-Yu Qiu, ${ }^{5}$ Yan-Shang Xiao, ${ }^{6}$ and Qun-Feng \\ $\mathrm{Lu}^{6, *}$ \\ ${ }^{1}$ Otolaryngology Head and Neck Surgery, Shanghai Children's Hospital, Shanghai Jiao Tong University, Shanghai, China \\ ${ }^{2}$ Comprehensive Management Department, Shanghai Children's Hospital, Shanghai Jiao Tong University, Shanghai, China \\ ${ }^{3}$ Gastroenterology Department, Shanghai Children's Hospital, Shanghai Jiao Tong University, Shanghai, China \\ ${ }^{4}$ Pneumology Department, Shanghai Children's Hospital, Shanghai Jiao Tong University, Shanghai, China \\ ${ }^{5}$ Neonatal Intensive Care Unit, Shanghai Children's Hospital, Shanghai Jiao Tong University, Shanghai, China \\ ${ }^{6}$ Nursing Department, Shanghai Children's Hospital, Shanghai Jiao Tong University, Shanghai, China \\ "Corresponding authors: Mei Gong, Comprehensive Management Department, Shanghai Children's Hospital, Shanghai Jiao Tong University, Shanghai, China. Tel/Fax: \\ +86-2162476336, E-mail: littlegrass1987@hotmail.com; Qun-Feng Lu, Nursing Department, Shanghai Children's Hospital, Shanghai Jiao Tong University, Shanghai, China. Tel: \\ +86-2152976239, Fax: +86-2152976067, E-mail: luqf@shchildren.com.cn
}

Received 2016 April 11; Revised 2016 June 16; Accepted 2016 June 30.

\begin{abstract}
Background: Establishing venous access is a challenging job for pediatric nurses, especially in the emergency department. Measures to ensure higher success rates on the first attempt are important to provide quality nursing care.

Objectives: To explore the effect of grade management on the success of establishing peripheral venous access in the child population of China and to analyze the factors influencing failed IV access on the first attempt.

Methods: This is an observational study on children aged 0 - 16 years old undergoing peripheral venous catheterization in a children's hospital. Patient information was collected before attempting each puncture. Logistic regression was used to identify independent factors for success prediction.

Results: A total of 1,016 subjects enrolled. The success rate of intravenous puncture on the first attempt was $86.02 \%$. This can be influenced by several factors, such as patient age, department, venous condition, and nurse experience. The success rate within two attempts was $96.85 \%$; the number of catheters used per IV attempt was 1.21.

Conclusions: To date, only a few studies have explored the success rate of peripheral IV catheterization in the pediatric population of China. Grade management of peripheral veins and pediatric nurses facilitated higher first-attempt success than in previously published reports. Failures of catheterization were multifactorial. The success rate of peripheral intravenous insertion in children can be improved through applying assistance devices or enhancing the venipuncture skills of pediatric nurses.
\end{abstract}

Keywords: Grade Management, Peripheral Venous Access, Children

\section{Background}

Peripheral intravenous (IV) catheterization is the most common nursing procedure in clinical practice, and it should be mastered by every registered nurse in China. Although this is a basic technique performed by nurses on a daily basis, establishing IV access is still a challenging job for many pediatric nurses because it is not easy to obtain IV access in children, especially in neonates. Some of the challenges are unique to this population.

There are several published studies addressing successful IV catheterization. A 2015 study reported a firstattempt success rate of $85 \%$ with a guidewire-associated peripheral venous catheter (GAPIV) versus $22 \%$ with the conventional peripheral venous catheter. A second attempt was required for $62 \%$ of patients with the conventional catheter compared to $15 \%$ with the GAPIV (1). IV placement can be challenging in pediatric patients; the failure rate can reach $47 \%$ on the first attempt, and $9 \%$ of patients require more than four attempts before peripheral IV access is obtained (2). The data from an emergency department reported a success rate of $67.8 \%$ on the first catheterization attempt in pediatric patients (3). Even when interventions such as venous imaging ultrasound are used, two or more attempts are still required for $15 \%$ of children to achieve access (4).

Multiple intravenous attempts cause pain, distress, anxiety, and even conflicts with patients. According to a report in China, 31\% of conflicts occurring in the outpatient department of the children's hospital were caused by failed catheterization attempts, out of which $27.3 \%$ occurred because of an unskilled puncture (5). Managing difficult venous access or applying instruments can reduce IV placement failure $(4,6,7)$. However, there is no objective data from a large sample of successful catheterization at- 
tempts and influencing factors in China, and guidelines or standard management methods for this issue are still lacking. Risk factors for first-attempt failures have not been taken into consideration, and there have been no large sample studies analyzing access difficulties.

\section{Objectives}

The purpose of this study was to detect the success rate of peripheral venous catheterization in Chinese pediatric patients after grade management and to further detect the risk factors for failed insertion on the first attempt.

\section{Methods}

\subsection{Study Design and Population}

This is a prospective, clinical observational study conducted between December, 2014 and June, 2015 in Shanghai children's hospital. This study involved pediatric inpatients who received peripheral venous catheterization and the pediatric nurses who established access for them. Quota sampling was used to enroll patients from the department of internal medicine, the department of surgery, and the pediatric intensive care unit. The patients' caregivers and nurses were informed of the purpose and contents of the study, and the nurses enrolled in data collections were trained in the methods and rules concerning the information tabulation. The following patients were excluded: outpatients, patients who needed central line access (defined by the treating physician), and patients or parents who refused to participate in the data collection. Based on the procedure designed by Field (8). and the overall staff conditions at our hospital, we outlined the common practice procedure for catheterization as follows: 1, the doctor determines whether to establish venous access or not; 2, the registered nurse performs IV placement; 3 , once placement fails, an evaluation is conducted again to determine whether the current nurse should continue the placement; if two attempts fail, members of the vein management group are called to attempt vascular access with or without a vein-imaging instrument. When venous access is difficult to establish, physicians should re-evaluate the need for an IV line and consider alternatives to IV therapy. The procedure mentioned in this study is in accordance with the standards defined by the ethics committee on human experimentation of Shanghai children's hospital (decision no. 2015R027-F01).

\subsection{Study Protocol}

Before placement, nurses were asked to document the patients' information, including two aspects: 1 , basic information (age, gender, weight, mental status, vein palpability, vein visibility, etc.) and 2, medical history (history of operation, radiotherapy, and chemotherapy). Postcatheterization data also included two aspects: 1 , infusion information (success on the first attempt [yes or no], site of failed/successful puncture, and number of total attempts); 2 , nurse information (age, professional title, nurse experience, educational history, self-reported IV placement ability, and whether they received related training [yes or no]). After the documentation was completed, an experienced nurse checked the information tabulation to ensure the integrity and accuracy of the information.

\subsection{Measures}

Yen's definition of successful IV catheterization is used in this study (9). An IV attempt begins from the time when the needle first touches the skin, and success is defined as an IV attempt in which the IV is placed well enough at the site that the infusion is able to be injected without swelling at the insertion site.

\subsection{Analysis}

SPSS 16.0 statistical software was used in this study. Demographic data was expressed as mean \pm standard deviation. Outcome frequency was examined using descriptive statistics. A chi-square test or Fisher's exact test was used for univariate analysis of the collected variables. An independent-samples t-test was used for analyzing differences between groups. Multivariate logistic regression was performed on the patients' demographic data and on the outcome of the first IV attempt to identify demographic risk factors for failed attempts. The adjusted odds ratios (ORs) of significant variables are reported with 95\% CIs. P values of 0.05 or less were considered significant.

\section{Results}

\subsection{Subjects' Characteristics}

There were 1,100 subjects who met the inclusion criteria; 70 were excluded because of refusal, and 14 because of incomplete data, leaving 1,016 patients for analysis. The mean age of patients was $3.28 \pm 3.32$ years. Of the patients, $610(60.0 \%)$ were male and $406(40.0 \%)$ were female. All the included nurses were female, and their mean age was 27.02 \pm 4.70 years (Table 1 ). 
Table 1. Demographics of the Subjects

\begin{tabular}{|c|c|}
\hline Characteristics & \\
\hline Patient age, $Y$ & $3.28 \pm 3.32$ \\
\hline Patient weight, kg & $15.87 \pm 13.25$ \\
\hline \multicolumn{2}{|l|}{ Gender } \\
\hline Male & $610(60.00)$ \\
\hline Female & $406(40.00)$ \\
\hline Department & No. (\%) of patients \\
\hline Internal medicine & $457(44.98)$ \\
\hline Department of surgery & $434(42.72)$ \\
\hline Intensive care unit & $125(12.30)$ \\
\hline \multicolumn{2}{|l|}{ Mental status } \\
\hline Good & $856(84.25)$ \\
\hline Common & $131(12.89)$ \\
\hline Poor & $29(2.85)$ \\
\hline \multicolumn{2}{|l|}{ Nurse experience (in years) } \\
\hline$\leq 1$ & $256(25.20)$ \\
\hline $2-4$ & $230(22.64)$ \\
\hline $5-9$ & $375(36.91)$ \\
\hline$\geq 10$ & $155(15.26)$ \\
\hline \multicolumn{2}{|l|}{ Nurse professional title } \\
\hline Nurse & $610(60.04)$ \\
\hline Senior nurse & $373(36.71)$ \\
\hline Nurse-in-charge & $33(3.25)$ \\
\hline \multicolumn{2}{|l|}{ Educational history } \\
\hline Technical secondary school & $48(4.72)$ \\
\hline Junior college & $650(63.98)$ \\
\hline Undergraduate or above & $318(31.30)$ \\
\hline
\end{tabular}

\subsection{Success Rates}

On the first attempt, the common locations were the dorsum of the hand $(606,59.65 \%)$, the dorsum of the foot $(109,10.73 \%)$ and the saphenous vein $(93,9.15 \%)$. A total of 776 (76.38\%) catheters from Becton, Dickinson and company were used. A safety catheter was used in 189 (18.60\%) subjects on the first attempt. According to the peripheral venous grading system in children designed at our hospital (Table 2), the veins of 381 (37.50\%) subjects were at level 0 , the veins of 364 (35.83\%) subjects were at level I, the veins of 198 (19.49\%) subjects were at level II, and the veins of 73 (7.19\%) subjects were at level III.

The success rate of the first attempt was $86.02 \%$, which decreased to $67.61 \%$ on the second attempt. Up to $13.98 \%$ of patients required two or more attempts to establish pe- ripheral venous access, and $4.53 \%$ of patients needed three or more attempts. This shows that 1.21 sticks on average are required per successful placement (Table 3). No patients in our sample underwent insertion of a peripherally-inserted central catheter (PICC), an intraosseous (IO) device, or a central venous catheter (CVC).

\subsection{Influencing Factors}

From the results of the univariate analysis on all variables, we can see that the success rate of the first attempt was influenced by patient age, weight, department, mental status, whether communications were effective or not, venous condition, and nurse age, experience, and professional title. A multivariate logistic regression was used to identify several independent risk factors for failed catheterization on the first attempt. The following patients were found to be at greater risk: younger children, patients admitted to the intensive care unit, patients with veins at level 0 or level I, and patients being served by nurses with experience ranging from two to four years or more than 10 years (Table 4 ).

\section{Discussion}

Peripheral IV access is routinely required for the diagnosis and treatment of many patients, and peripheral IV placement technique should be mastered by every registered nurse in China. However, there is still little information on the success rate of peripheral IV insertion in pediatric hospitals. Multiple IV attempts not only compromise the patients' trust and confidence in the nursing staff and/or the hospital but also cause pain and complications for patients. For these reasons, new technology for peripheral IV catheterization has been applied to improve success rates and to avoid the problems associated with placement $(7,10-12)$. According to the reports, the success rate of peripheral IV catheterization with the traditional method was between $33 \%$ and $70.6 \%$, whereas the ultrasound-guided peripheral IV access resulted in a success rate of $80.5 \%$ - $97 \%(11,12)$. The ultrasound-guided peripheral IV access program was also reported to improve the placement of central venous catheters (13).

Our results indicate an $86.02 \%$ success rate on the first attempt at freehand peripheral IV placement, which decreases to $67.61 \%$ on the second attempt. Therefore, when the first attempt fails, a new evaluation of the patient should be performed, and whether to adjust the performer should be considered. We made a guideline by which only two insertion attempts can be placed in the same subject by any one of the nurses. In emergency situations, successful peripheral IV access is achieved within two attempts in 
Table 2. Intravenous Access Scoring in Children ${ }^{\mathrm{a}}$

\begin{tabular}{lccc}
\hline Content & $\mathbf{0}$ & $\mathbf{1}$ & $\mathbf{2}$ \\
\hline Age, $\mathbf{y}$ & $\geq 3$ & $1-3$ & $\leq 1$ \\
Combination & Good & Average & Poor visible applied tourniquets \\
Visible & Visible & Vague applied tourniquets & No palpable applied tourniquets \\
Palpable & Palpable & Vague applied tourniquets & Poor \\
\hline Circulation & Good & Average & \\
\hline
\end{tabular}

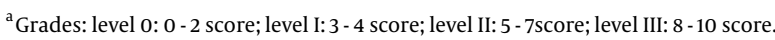

Table 3. Attempt Results

\begin{tabular}{|c|c|c|}
\hline Results & $\mathbf{N}$ & Rate, \% \\
\hline Success/failure on the first attempt & $874 / 142$ & $86.02 / 13.98$ \\
\hline Success/failure on the second attempt & $96 / 46$ & $67.61 / 32.39$ \\
\hline Success within two attempts & 970 & 95.47 \\
\hline Attempt count $\geq 3$ times & 46 & 4.53 \\
\hline Total no. of attempts & \multicolumn{2}{|c|}{1,226} \\
\hline Catheters per IV attempt & \multicolumn{2}{|c|}{1.21} \\
\hline
\end{tabular}

Table 4. Influencing Factors

\begin{tabular}{|c|c|c|c|c|c|}
\hline Variable & Control Group & Comparison Group & OR & 95\% CI & P Value \\
\hline Patient age & Continuous variables & & 1.124 & $1.043-1.220$ & 0.003 \\
\hline \multirow{2}{*}{ Department } & ICU & Internal medicine & 2.890 & $1.584-5.265$ & 0.001 \\
\hline & & Department of surgery & 2.206 & $1.154-4.210$ & 0.015 \\
\hline Vein level & Rank variable & (level 0, I, II, III) & 2.305 & $1.827-2.913$ & $<0.001$ \\
\hline \multirow{3}{*}{ Nurse experience, $y$} & $\geq 10$ & $\leq 1$ & 1.256 & $0.639-2.440$ & 0.517 \\
\hline & & $2-4$ & 1.344 & $0.723-2.463$ & 0.350 \\
\hline & & 5-9 & 2.347 & $1.325-4.167$ & 0.004 \\
\hline
\end{tabular}

$92 \%$ of adults (14) and in $86 \%$ of children (15). The success rate within two attempts is $95.47 \%$ in this study, which is higher than that in the reports mentioned above, meaning that $4.53 \%$ of patients require three or more attempts before success. Under these circumstances, vein management group members should be called to perform the insertion.

Several studies have attempted to predict the risk factors associated with difficult venous access. Obesity, diabetes mellitus, dehydration, and an unskilled performer may affect the success rate of the first attempt $(8,16)$. In our study, univariate analysis of 13 variables revealed that only nine factors (patient age, weight, department, mental status, whether communications are effective or not, venous condition, and nurse age, experience, and professional ti- tle) are significantly associated with the first-attempt success rate of peripheral IV access. In order to further explore the risk factors for failed IV insertion on the first attempt, we define whether success or failure on the first attempt is a dependent variable $(1=$ yes, $0=$ no), define the nine factors as independent variables, and fit the non-conditional logistic regression analysis. Logistic regression analysis of nine proposed predictor variables reveals that only four factors (patient age, department, and venous condition and nurse experience) are significantly associated with the first-attempt success rate of peripheral IV access (Table 4). The average age in the success group on the first attempt is $3.46 \pm 3.18$, whereas it is $2.51 \pm 2.93$ in the failed group $(\mathrm{P}<$ 0.001). A history of previous stays in the intensive care unit (ICU) has a greater association with failed peripheral IV in- 
sertion than a history of general ward visits $\left(\chi^{2}=17.632, \mathrm{P}<\right.$ 0.001 ). This demonstrates a higher success rate in patients with good venous condition (level 0, level I) (95\% CI: 1.816 2.913, $\mathrm{P}<0.001$ ).

Among the six operator (nurse) variables evaluated, only nurse experience proved to be statistically associated with peripheral IV placement.

It is widely assumed that nurses with more experience in placement can achieve a higher success rate. However, contrary to our expectations, nurses with between five and nine years of experience had the highest $(89.07 \%)$ success rate in our study, higher than that of the nurses with less than one year (87.89\%), two to four years (84.78\%), and greater than 10 years $(77.42 \%)(\mathrm{P}<0.01)$ of experience. This may be related to the fact that experienced nurses often perform the difficult venous access attempts and assume responsibility for venous consultation (Figure $1 \mathrm{~A}$ ). In this study, the opportunities of the nursing staff to establish IV access varied according to their experience. It is an effective strategy to empower each nurse to do meaningful work and to improve their organizational commitments. There is a positive relationship between nurse experience and first-attempt success rates. Nurse managers should consider how to apply this method, as well as measures for improvement.

According to the report, higher levels of self-reported IV placement ability are always associated with a higher success rate of IV insertion (17). In this study, although the nurses who self-identified as skilled in IV placement ability achieved the highest success rate (86.59\%), higher than those of the other two groups (Figure 1B), this difference was not found to be statistically significant. This indicates that nurses may have inaccurate self-assessment abilities in this context, especially less-experienced nurses. Therefore, we propose objective variables for more accurate evaluation of nurse venipuncture skill.

The management of peripheral IV insertion is not standardized (18). Identifying the potentially difficult venous access cases at an early stage is important for nurses to adjust their approach and staff, which can contribute to improving the success rate. There are many methods to manage peripheral IV insertion, such as anesthetizing the peripheral IV site to reduce patient pain and discomfort (1921) using an assistive device to improve vein visualization to achieve a higher success rate (22-26) applying diagnostic tests to confirm correct intravascular placement (27) and local warming (28). Considering the fact that each registered nurse can establish peripheral IV access for pediatric patients, we proposed grades to measure the venipuncture skills of pediatric nurses (Figure 2). We assigned appropriately skilled nurses according to the peripheral vein grade of each case. Each nurse should be examined by experi-
A

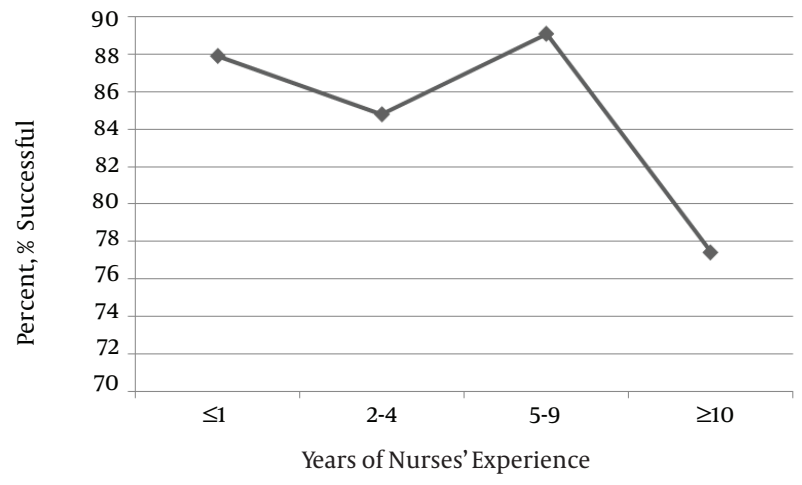

B

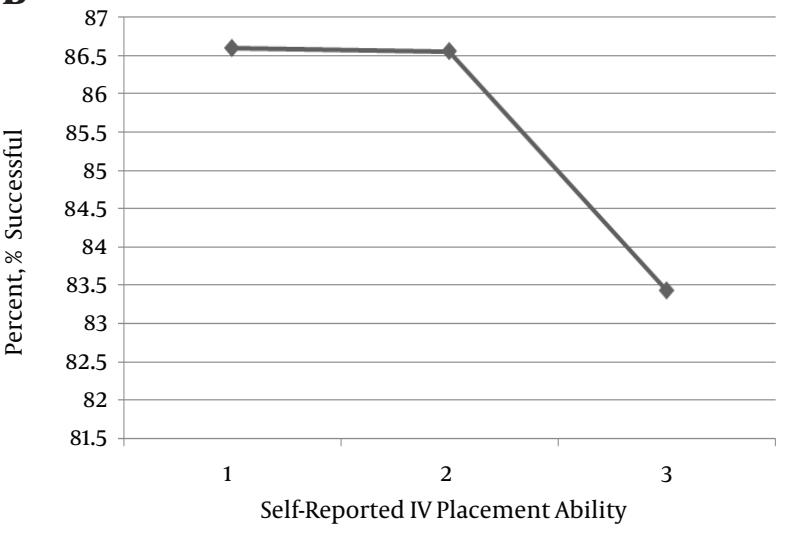

Figure 1. A, Relationship between nurse experience and success rate; B, relationship between nurse self-reported IV placement ability and success rate (self-reported IV placement ability on a scale of 1 for skilled to 3 for not at all skilled)

enced nurses from the vein management group and by the head-nurse to evaluate their success rate for first attempts at insertion on a given grade of vein. When the success rate is above $90 \%$, a nurse should be considered qualified to perform insertions for the next level of vein.

\subsection{Limitations}

The major limitation of this study is that it is an observational study. Although the sample size is sufficient, selection bias may have occurred due to the fact that some patients refused to participate, although refusing to participate would likely be random. Meanwhile, all the subjects were enrolled in a single pediatric hospital. Therefore, more studies from multiple centers in this field using a prospective, randomized study design are recommended. The second limitation is that patient diagnosis, skin condition, and nutrition condition were not analyzed for risk factors in this study. In future studies, these factors should be internalized in the study design for analysis. 

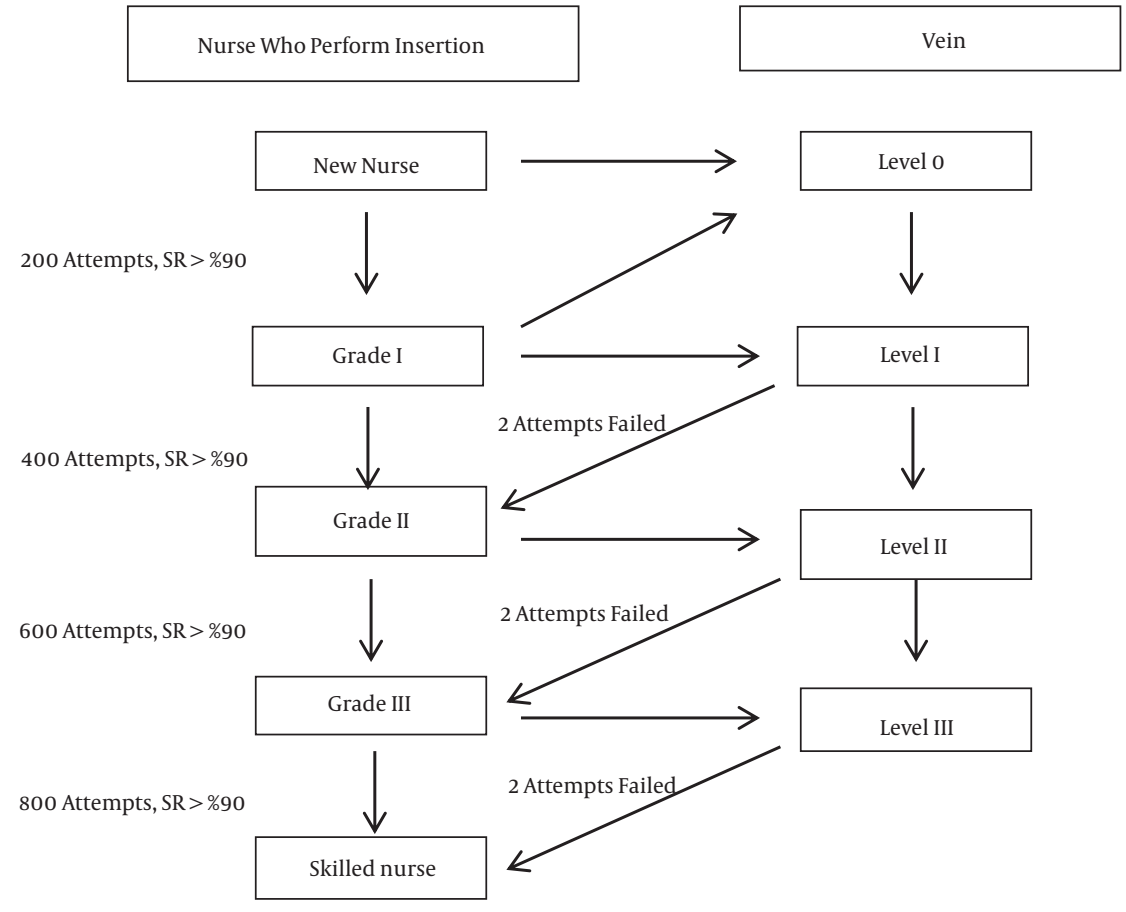

Figure 2. Management of Venipuncture in Children (SR = Success Rate)

\subsection{Conclusions}

The findings of this study provide important information about the success rate of peripheral IV access and risk factors for failed placement on the first attempt in pediatric patients in China.

Grade management of veins and improving nurse venipuncture skill are feasible measures in pediatric patients. In our investigation, the success rate of the first attempt was higher than in previously reported data, but it significantly decreased on the second attempt. This illustrates that grade management can be used to guide the ordering of nurses to achieve timely vascular access and a high success rate on the first attempt. This study also establishes that management measures should be revised in the future for standard peripheral IV insertion in pediatric patients, especially when multiple intravenous attempts are needed. The risk factors for failed access on the first attempt include patient age, department, and venous condition and nurse experience, which should be considered before IV insertion.

\section{Acknowledgments}

The authors sincerely thank Jiangjiang Xu for guidance in data collection and statistical analysis and the education nurses from every department for assisting with training in the method of inputting patients' information.

\section{Footnotes}

Authors' Contribution: Mei Gong and Qun-Feng Lu designed this study. Ya-Min Yan formulated the information table, performed the survey, and drafted the article. Dan Li and Ye Huang participated in training the information collector and collecting the data. Ai-Qiu Li and Yan-Shang Xiao participated in formulating the management methods and drafting the article. Mei Gong and Jia-Yu Qiu participated in evaluating vein conditions. All authors have read and approved this article.

Ethical Approval: The procedure mentioned in this study was in accordance with the standards of the ethics committee on human experimentation of Shanghai children's hospital (Decision no. 2015R027-F01).

Funding/Support: This work was supported by the Youth program of the Shanghai municipal commission of health and family planning (No. 20134Y090) and the program of the Shanghai Shenkang hospital development center (No. SHDC2014616). 


\section{References}

1. Chiricolo G, Balk A, Raio C, Wen W, Mihailos A, Ayala S. Higher success rates and satisfaction in difficult venous access patients with a guide wire-associated peripheral venous catheter. Am J Emerg Med. 2015;33(12):1742-4. doi: 10.1016/j.ajem.2015.08.005. [PubMed: 26458532].

2. Lininger RA. Pediatric peripheral i.v. insertion success rates. Pediatr Nurs. 2003;29(5):351-4. [PubMed:14651305].

3. Riker MW, Kennedy C, Winfrey BS, Yen K, Dowd MD. Validation and refinement of the difficult intravenous access score: a clinical prediction rule for identifying children with difficult intravenous access. Acad Emerg Med. 2011;18(11):1129-34. doi:10.1111/j.1553-2712.2011.01205.x. [PubMed: 22092893].

4. Katsogridakis YL, Seshadri R, Sullivan C, Waltzman ML. Veinlite transillumination in the pediatric emergency department: a therapeutic interventional trial. Pediatr Emerg Care. 2008;24(2):83-8. doi: 10.1097/PEC.0b013e318163db5f. [PubMed: 18277843].

5. Wang X, Zhang L, Liu X. The cause and management of nurse-patient conflicts in pediatric outpatients. J Qilu Nurs. 2009;15:83-4.

6. Sebbane M, Claret PG, Lefebvre S, Mercier G, Rubenovitch J, Jreige $\mathrm{R}$, et al. Predicting peripheral venous access difficulty in the emergency department using body mass index and a clinical evaluation of venous accessibility. J Emerg Med. 2013;44(2):299-305. doi: 10.1016/j.jemermed.2012.07.051. [PubMed: 22981661].

7. Kumar A, Chuan A. Ultrasound guided vascular access: efficacy and safety. Best Pract Res Clin Anaesthesiol. 2009;23(3):299-311. [PubMed: 19862889].

8. Fields JM, Piela NE, Au AK, Ku BS. Risk factors associated with difficult venous access in adult ED patients. Am J Emerg Med. 2014;32(10):117982. doi: 10.1016/j.ajem.2014.07.008. [PubMed: 25171796].

9. Yen K, Riegert A, Gorelick MH. Derivation of the DIVA score: a clinical prediction rule for the identification of children with difficult intravenous access. Pediatr Emerg Care. 2008;24(3):143-7. doi: 10.1097/PEC.ob013e3181666f32. [PubMed: 18347490].

10. Witting MD, Schenkel SM, Lawner BJ, Euerle BD. Effects of vein width and depth on ultrasound-guided peripheral intravenous success rates. J Emerg Med. 2010;39(1):70-5. doi: 10.1016/j.jemermed.2009.01.003. [PubMed:19272730].

11. Costantino TG, Parikh AK, Satz WA, Fojtik JP. Ultrasonographyguided peripheral intravenous access versus traditional approaches in patients with difficult intravenous access. Ann Emerg Med. 2005;46(5):456-61. doi: 10.1016/j.annemergmed.2004.12.026. [PubMed: 16271677].

12. Bauman M, Braude D, Crandall C. Ultrasound-guidance vs. standard technique in difficult vascular access patients by ED technicians. Am J Emerg Med. 2009;27(2):135-40. doi: 10.1016/j.ajem.2008.02.005. [PubMed: 19371518].

13. Shokoohi H, Boniface K, McCarthy M, Khedir Al-tiae T, Sattarian $\mathrm{M}$, Ding $\mathrm{R}$, et al. Ultrasound-guided peripheral intravenous access program is associated with a marked reduction in central venous catheter use in noncritically ill emergency department patients. Ann Emerg Med. 2013;61(2):198-203. doi: 10.1016/j.annemergmed.2012.09.016. [PubMed: 23141920].

14. Lapostolle F, Catineau J, Garrigue B, Monmarteau V, Houssaye T, Vecci I, et al. Prospective evaluation of peripheral venous access dif- ficulty in emergency care. Intensive Care Med. 2007;33(8):1452-7. doi: 10.1007/s00134-007-0634-y. [PubMed: 17554524].

15. Black KJ, Pusic MV, Harmidy D, McGillivray D. Pediatric intravenous insertion in the emergency department: bevel up or bevel down?.Pediatr Emerg Care. 2005;21(11):707-11. [PubMed:16280942].

16. O'Neill MB, Dillane M, Hanipah NF. Validating the difficult intravenous access clinical prediction rule. Pediatr Emerg Care. 2012;28(12):1314-6. doi: 10.1097/PEC.0b013e3182768bc9. [PubMed: 23187988].

17. Jacobson AF, Winslow EH. Variables influencing intravenous catheter insertion difficulty and failure: an analysis of 339 intravenous catheter insertions. Heart Lung. 2005;34(5):345-59. doi: 10.1016/j.hrtlng.2005.04.002. [PubMed:16157191].

18. Strauss JM, Denk A. [The difficult venous access]. Anasthesiol Intensivmed Notfallmed Schmerzther. 2013;48(4):258-63. doi: 10.1055/s-00331343761. [PubMed: 23633258].

19. McNelis KA. Intradermal bacteriostatic $0.9 \%$ sodium chloride containing the preservative benzyl alcohol compared with intradermal lidocaine hydrochloride $1 \%$ for attenuation of intravenous cannulation pain. AANA J. 1998;66(6):583-5. [PubMed: 10488265].

20. Windle PE, Kwan ML, Warwick H, Sibayan A, Espiritu C, Vergara J. Comparison of bacteriostatic normal saline and lidocaine used as intradermal anesthesia for the placement of intravenous lines. JPerianesth Nurs. 2006;21(4):251-8. doi: 10.1016/j.jopan.2006.05.007. [PubMed: 16935736].

21. Burke SD, Vercler SJ, Bye RO, Desmond PC, Rees YW. Local anesthesia before IV catheterization. Am J Nurs. 2011;111(2):40-5. doi: 10.1097/01.NAJ.0000394291.40330.3c. [PubMed: 21270583] quiz 46-7.

22. Peterson KA, Phillips AL, Truemper E, Agrawal S. Does the use of an assistive device by nurses impact peripheral intravenous catheter insertion success in children?. J Pediatr Nurs. 2012;27(2):134-43. doi: 10.1016/j.pedn.2010.10.009. [PubMed: 22341192].

23. John JM. Transillumination for vascular access: old concept, new technology. Paediatr Anaesth. 2007;17(2):197-8. doi: 10.1111/j.14609592.2006.02061.x. [PubMed: 17238901].

24. Krueger A. Need help finding a vein?. Nursing. 2007;37(6):39-41. doi: 10.1097/01.NURSE.0000271810.56917.a0. [PubMed: 17538434].

25. Miyake RK, Zeman HD, Duarte FH, Kikuchi R, Ramacciotti E, Lovhoiden G, et al. Vein imaging: a new method of near infrared imaging, where a processed image is projected onto the skin for the enhancement of vein treatment. Dermatol Surg. 2006;32(8):1031-8. doi: 10.1111/j.1524-4725.2006.32226.x. [PubMed:16918565].

26. Goren A, Laufer J, Yativ N, Kuint J, Ben Ackon M, Rubinshtein M, et al. Transillumination of the palm for venipuncture in infants. Pediatr Emerg Care. 2001;17(2):130-1. [PubMed:11334094].

27. Keidan I, Sidi A, Ben-Menachem E, Derazne E, Berkenstadt H. A simple diagnostic test to confirm correct intravascular placement of peripheral catheters in order to avoid extravasation. J Clin Anesth. 2015;27(7):585-8. doi:10.1016/j.jclinane.2015.07.004. [PubMed: 26286133].

28. Lenhardt R, Seybold T, Kimberger O, Stoiser B, Sessler DI. Local warming and insertion of peripheral venous cannulas: single blinded prospective randomised controlled trial and single blinded randomised crossover trial. BMJ. 2002;325(7361):409-10. [PubMed: 12193353]. 